\title{
A generalized Derjaguin approximation for electrical-double-layer interactions at arbitrary separations
}

\author{
Ory Schnitzer \\ Department of Mathematics, Imperial College London, \\ South Kensington Campus, London SW7 2AZ, United Kingdom \\ Matvey Morozov \\ Department of Mathematics, Technion - Israel Institute of Technology, Haifa 32000, Israel
}

\begin{abstract}
Derjaguin's approximation provides the electrical-double-layer interaction force between two arbitrary convex surfaces as the product of the corresponding one-dimensional parallel-plate interaction potential and an effective radius $R$ (function of the radii of curvature and relative orientation of the two surfaces at minimum separation). The approximation holds when both the Debye length $1 / \kappa$ and minimum separation $h$ are small compared to $R$. We show here that a simple transformation,

$$
R \Rightarrow[R] \sqrt{\frac{\left[K_{1}\right]\left[K_{2}\right]}{K_{1} K_{2}}},
$$

yields an approximation uniformly valid for arbitrary separations $h$; here $K_{i}$ is the Gaussian curvature of particle $i$ at minimum separation, and [ $\cdot]$ is an operator which adds $h / 2$ to all radii of curvature present in the expression on which it acts. We derive this result in two steps. First, we extend the two-dimensional ray-theory analysis of Schnitzer [Physical Review E, 91022307 2015], valid for $\kappa h, \kappa R \gg 1$, to three dimensions. Using this approach we obtain a general closed form expression for the force by matching nonlinear diffuse-charge boundary layers with a WKBJ description of the bulk potential, and subsequent integration via Laplace's method of the traction over the medial surface generated by all spheres maximally inscribed between the two surfaces. Second, we exploit the existence of an overlap domain, $1 \ll \kappa h \ll \kappa R$, where both the ray-theory and the Derjaguin approximations hold, to systematically form the generalized mapping. The validity of the result is demonstrated by comparison with numerical computations.
\end{abstract}




\section{INTRODUCTION}

Interfaces between solid surfaces and electrolyte solutions are characterised by charge separation [1], i.e. the formation of "electrical double layers" (EDL). The solid side spontaneously acquires surface charge, while on the electrolyte side a balance between electrostatic forces and diffusion results in the formation of a screening ionic cloud ("diffuse-charge", or "Debye" layer), where counter-ions are in excess. Two basic quantities describe a diffusecharge layer: the (typically nano-metric) Debye length scale on which space-charge density attenuates towards the electro-neutral bulk, and the zeta potential, the voltage drop across the layer. EDL play a key role in numerous physical phenomena: They largely determine the stability, and the effective conductive, optical and rheological properties, of particulate colloidal solutions and emulsions [2]; enable a wide variety of electrokinetic phenomena with colloidal particles $[1,3]$, in micro-channels [4-6], and in porous medium [7]; affect current passage in electrochemical configurations [8]; and are responsible for ion-selectivity of nano-pore membranes and nano-channels [9].

A fundamental consequence of charge separation is the EDL interaction force acting between two diffusely screened charged surfaces. In many scenarios even a slight diffuselayer overlap gives rise to a force which is appreciable, in the sense that it is comparable with other forces present in the system [10]. For example, a balance with van der Waals attraction regulates the stability of colloidal solutions to aggregation [11]; a balance with gravity enables levitation of a micron-sized particle above an electrode $[12,13]$; and a balance with various coiling mechanisms determines the conformation of DNA molecules in solution $[14,15]$. EDL interactions are also important in various environmental applications such as

coal flotation [16], biological phenomena [17-20], and interpreting AFM measurements in conducting liquids for imaging [21] or in fundamental surface-physics studies [22, 23].

There is a huge body of literature devoted to the theoretical modelling of EDL interactions, both classic and contemporary. Many of these works are based on a continuum-level description [largely the Poisson-Boltzmann (PB) formulation and generalisations thereof], while others are based on more detailed descriptions such as density-functional theory and molecular-dynamics simulations [24]. While the most tractable configuration for analysis and computation is the one-dimensional parallel-plate system, all of the applications mentioned in the preceding paragraph necessarily involve curved surfaces. For this reason, Derjaguin's 
(or Deryagin's) formula [11, 25, 26], which provides an approximate mapping from one to two or three dimensions, has become the paramount theoretical tool for studying and predicting the effects of EDL interactions [27, 28].

In mathematical terms, Derjaguin's approximation provides the interaction force between two arbitrarily shaped convex surfaces at minimum separation $h^{*}$ as (henceforth, all dimensional quantities are decorated by an asterisk)

$$
F^{*}\left(h^{*}\right) \sim 2 \pi R^{*} \int_{h^{*}}^{\infty} \Pi^{*}\left(s^{*}\right) d s^{*}
$$

where $\Pi^{*}\left(h^{*}\right)$ is the force per unit area of the corresponding parallel plate system at separation $h^{*}$, and $R^{*}$ is the "effective radius", a geometric factor depending only on the intrinsic geometry of the surfaces near minimum separation; the force acts along the minimumdistance line connecting the surfaces, with $F^{*}>0$ corresponding to a repulsive interaction. For spheres of radii $R_{1}^{*}$ and $R_{2}^{*}$, the effective radius is

$$
\frac{1}{R^{*}}=\frac{1}{R_{1}^{*}}+\frac{1}{R_{2}^{*}}
$$

while the generalisation to entirely arbitrary convex surfaces is [26]

$$
\frac{1}{R^{* 2}}=\left(\frac{1}{R_{11}^{*}}+\frac{1}{R_{21}^{*}}\right)\left(\frac{1}{R_{12}^{*}}+\frac{1}{R_{22}^{*}}\right)+\left(\frac{1}{R_{11}^{*}}-\frac{1}{R_{12}^{*}}\right)\left(\frac{1}{R_{21}^{*}}-\frac{1}{R_{22}^{*}}\right) \sin ^{2} \phi,
$$

where $R_{i j}^{*}$ denotes the principal radii of curvature $j=1,2$ of particle $i=1,2$, and $\phi$ is the angle between the principal curvature bases, all evaluated at minimum separation. Derjaguin's approximation is appropriate when both the gap separation $h^{*}$ and the Debye length scale $1 / \kappa^{*}$ are small compared to the radii of curvature characterising the gap geometry, say $R^{*}[26]$. For later reference, Fig. 1 depicts this domain of validity in the parameter space $\left(\kappa^{*} R^{*}, \kappa^{*} h^{*}\right)$. In light of the condition $h^{*} \ll R^{*}$, Derjaguin's mapping is also known as the "proximity-force approximation".

The long-standing availability of Derjaguin's approximation has guided further theoretical studies in two directions. The first is to improve our understanding of the one-dimensional configuration, via modified ion-transport and surface-chemistry models, exact and approximate solutions to continuum equations, and comparison with highly detailed molecular-level simulations. The second is to either improve on the approximation provided by Derjaguin's mapping or to go beyond its above-mentioned domain of validity; the present contribution is of the latter type. 


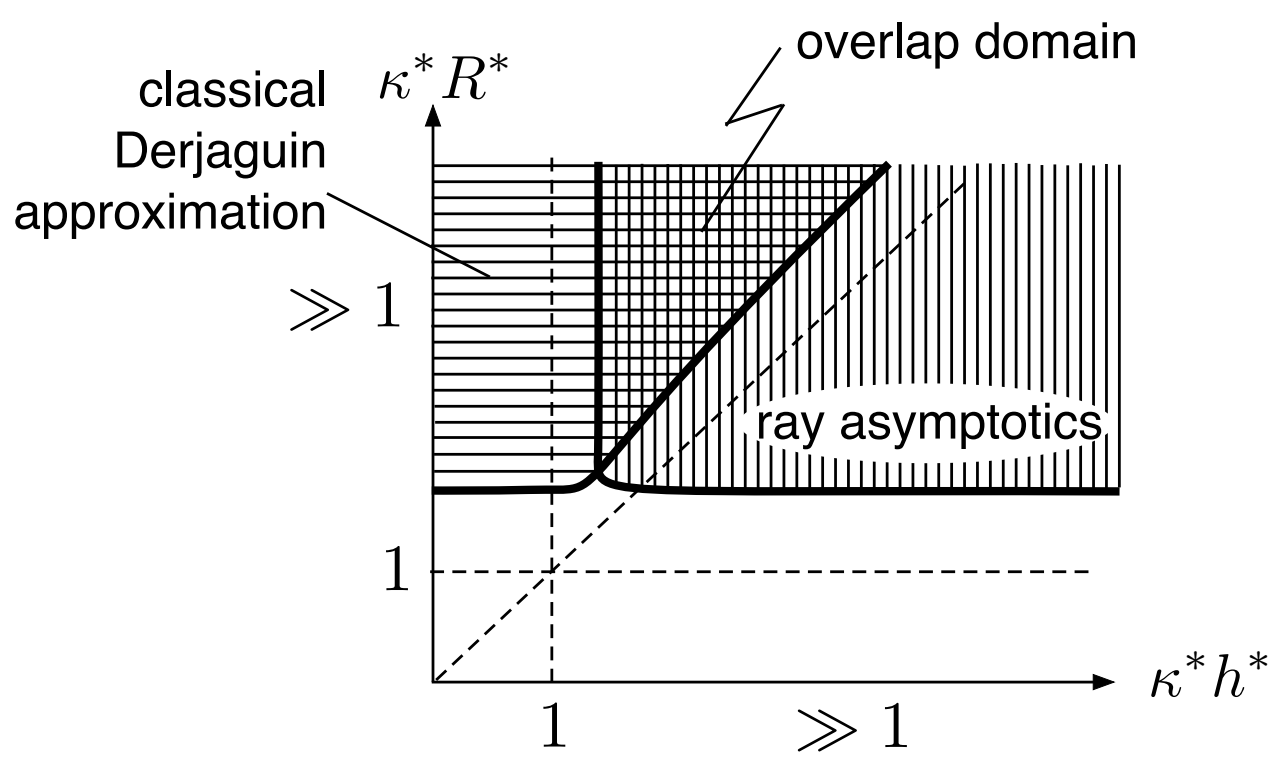

FIG. 1: The transformation (4) extends the domain of validity of Derjaguin's approximation to all separations $h^{*}$, the only remaining condition being $\kappa^{*} R^{*} \gg 1$. We first derive a novel closed-form formula valid for $\kappa^{*} h^{*} \gg 1$, building on the ray-theory approach of Ref. 29. We then exploit the depicted overlap of this approximation with the classical Derjaguin approximation towards forming a uniformly valid expression.

Rigorous treatments in two or three dimensions have been largely limited to idealised geometries. These include "exact" numerical simulations of the nonlinear PB formulation for identical and differing spheres [30-32], and numerical [33], analytic [34], and approximate [35] solutions of the linearised PB equation limited to low potentials. An alternative approximate approach known as the superposition approximation is valid for $\kappa^{*} h^{*}, \kappa^{*} R^{*} \gg 1$. In that domain, the potential distribution is accurately given by superposing the distributions associated with isolated surfaces. This simplification gives rise to several schemes, both numerical and analytical. In the low-voltage régime, the single-particle distributions may be found analytically by solving the linear PB equation for ideal geometries, typically spheres. Beyond low voltages, linear solutions of the latter type — now describing the almost electro-neutral bulk — may be joined with a one-dimensional solutions of the nonlinear diffuse layers [36, 37]. As it stands, the superposition approximation relies on having exact solutions to either the linear or nonlinear PB equation whereby a simple generalisation to arbitrary geometries appears intractable. 
In this paper, we show that a surprisingly simple generalisation of Derjaguin's approximation renders it uniformly valid for all separations $h^{*}$, the only remaining condition being $\kappa^{*} R^{*} \gg 1$. We find that, for arbitrary three dimensional convex surfaces, the requisite generalisation is obtained by modifying the effective radius (3) according to the rule

$$
R^{*} \Rightarrow\left[R^{*}\right]_{*} \sqrt{\frac{\left[K_{1}^{*}\right]_{*}\left[K_{2}^{*}\right]_{*}}{K_{1}^{*} K_{2}^{*}}},
$$

where $K_{1}^{*}$ and $K_{2}^{*}$ are the Gaussian curvatures of the two surfaces at minimum separation, and $[\cdot]_{*}$ represents an operator which adds $h^{*} / 2$ to all radii of curvatures. For example, $1 / K_{1}^{*}=R_{11}^{*} R_{12}^{*}$ becomes $1 /\left[K_{1}^{*}\right]_{*}=\left(R_{11}^{*}+h^{*} / 2\right)\left(R_{12}^{*}+h^{*} / 2\right)$. For simplicity we shall derive the above result in the familiar nonlinear PB framework appropriate for dilute electrolytes (assuming a symmetric binary electrolyte). In $\S \mathrm{VI}$, however, we shall assert that (4) holds for a more general class of models; this is consistent with the purely geometric form of the generalisation.

We obtain this result in two steps. The first consists of analysing the nonlinear PB formulation in the limit $\kappa^{*} R^{*} \gg 1$ and $\kappa^{*} h^{*} \gg 1$ (see Fig. 1). To this end, we extend the ray-theory approach one of us recently put forward in two dimensions [29] to three dimensions. In sharp contrast to the previous investigations of this limit $[36,37]$, the ray-theory approach yields a simple closed-form approximation valid for arbitrary three-dimensional convex surfaces. Thus instead of relying on exact solutions describing the bulk domain, the ray approach employs a WKBJ ansatz for this domain. The latter expansion is determined using analogies with ray-optics, and applying a rigorous matching condition with the nonlinear diffuse-charge boundary layers. But the crux of the method lies in the asymptotic evaluation of the interaction force. The surface over which stress is integrated is deformed to the "medial surface" generated by the centers of all spheres maximally inscribed between the two surfaces; the stress decays exponentially over this surface, and this allows us to use Laplace's method to extract the leading-order force in simple analytic form. The remaining step of the derivation relies on the observation that the validity régimes of the Derjaguin and the new ray-theory approximations overlap (see Fig. 1). We exploit this towards systematically combining the two approximations.

The rest of this paper is structured as follows. In $\S I I$ we formulate the problem of calculating the EDL interaction force. Following Ref. 29, in $\S$ III we asymptotically address the limit where $\kappa^{*} R^{*}$ and $\kappa^{*} h^{*}$ are both $\gg 1$; this yields a new closed-form approximation for 
arbitrary three-dimensional convex surfaces. In $\S I V$ we combine the latter with Derjaguin's approximation thereby obtaining a generalized Derjaguin approximation uniformly valid for all separations $h^{*}$. In $\S \mathrm{V}$ we compare the new formulae with numerical solutions of the nonlinear PB formulation. Concluding remarks are given in $\S \mathrm{VI}$.

\section{PROBLEM FORMULATION}

Consider two convex particles of otherwise arbitrary shape, their boundaries separated by a minimal distance $h^{*}$. The particles are suspended in an unbounded binary symmetric electrolyte of valency $\pm \mathcal{Z}$, far-field concentration $c^{*}$, and dielectric constant $\epsilon^{*}$. For simplicity we shall refer to either of the following canonical surface-charge models. (i) "fixed potential": a voltage $\varphi^{*} \psi_{i}$ is prescribed between the surface of particle $i=1,2$ and the far-field potential. (ii) "fixed charge": a uniform surface-charge density $\left(\epsilon^{*} \kappa^{*} \varphi^{*}\right) \sigma_{i}$ is prescribed. As for the Derjaguin approximation, the results we shall derive hold under more general charging conditions. We also disregard the electric displacement within the solids, a negligible effect in the parameter domain we are concerned with (see §VI). It is assumed that the positions and orientations of the particles are fixed, that the fluid is at rest, and that the solid-electrolyte system is in a state of equilibrium. The ionic distributions are therefore Boltzmann distributed. The goal outcome of the formulation below is the interaction force acting on the particles.

Henceforth, we shall employ a dimensionless formulation where ionic concentrations are normalized by $c^{*}$; potentials by the thermal voltage $\varphi^{*}=k^{*} T^{*} / \mathcal{Z} e^{*}, k^{*} T^{*}$ being the Boltzmann temperature and $e^{*}$ the electron-charge magnitude; lengths by $a^{*}$, a typical lengthscale characteristic of the particles; and pressure and stress by $\epsilon^{*}\left(\varphi^{*} / a^{*}\right)^{2}$. The corresponding dimensionless ionic concentrations, electric potential, and hydrostatic pressure, are respectively denoted by $c^{ \pm}, \varphi$, and $p$. The dimensionless minimal separation is denoted by

$$
h=\frac{h^{*}}{a^{*}},
$$

and the dimensionless Debye length is denoted by

$$
\delta=\frac{1}{\kappa^{*} a^{*}}, \quad \text { where } \quad \kappa^{* 2}=\frac{2 \mathcal{Z} e^{*} c^{*}}{\epsilon^{*} \varphi^{*}} .
$$

Choosing $\varphi$ to decay at large distances, and substituting Boltzmann's distribution $c^{ \pm}=$ 
$e^{\mp \varphi}$ into Poisson's equation, we find the nonlinear PB equation

$$
\delta^{2} \nabla^{2} \varphi=\sinh \varphi .
$$

As discussed above, on the particle boundaries $(i=1,2)$ we have one of the two conditions

$$
\varphi=\psi_{i} \quad \text { or } \quad \frac{\partial \varphi}{\partial n}=-\delta^{-1} \sigma_{i},
$$

respectively corresponding to the cases of fixed potential or fixed charge.

Given $\varphi$, the force on particle $i$ is found by integrating the stress on the particle boundary $S_{i}$. Thus the force (normalised by $\epsilon^{*} \varphi^{* 2}$ ) is provided by the integral

$$
\mathbf{f}_{i}=\oint_{S_{i}} \hat{\mathbf{n}} \cdot \mathrm{T} d A,
$$

where $\hat{\mathbf{n}}$ is the local outer normal to $S_{i}$, and T is the dimensionless stress tensor,

$$
\mathrm{T}=-p \mathbf{I}+\boldsymbol{\nabla} \varphi \boldsymbol{\nabla} \varphi-\frac{1}{2}|\boldsymbol{\nabla} \varphi|^{2} \mathbf{I},
$$

where I denotes the unity tensor. The stress tensor (10) combines a hydrostatic-pressure contribution, and the electrical (Maxwell) stress tensor. The hydrostatic pressure distribution is determined in terms of $\varphi$ from the mechanical equilibrium equation $\boldsymbol{\nabla} p=\nabla^{2} \varphi \boldsymbol{\nabla} \varphi$. Integrating the latter equation in conjunction with (7), and choosing the pressure to decay at large distances, one finds

$$
p=\delta^{-2}(\cosh \varphi-1) .
$$

Note that mechanical equilibrium can equivalently be stated as $\boldsymbol{\nabla} \cdot \boldsymbol{T}=0$. Thus the forces on the particles are equal in magnitude and opposite in sign, and the integration boundary in (9) can be arbitrarily deformed outward from the particle boundary as long as it does not intersect or encloses the second particle.

\section{THE LIMIT $\delta \ll 1$ AND $\delta \ll h$ : RAY-THEORY APPROACH}

In this section we analyse the asymptotic limit where the Debye length is small compared to both the separation and the characteristic particle size. In the dimensionless notation of $\S I$ II, these conditions read $\delta \ll 1$ and $\delta \ll h$. Note that we do not require $h$ to be small. Where possible, we shall follow the corresponding analysis of the simpler two-dimensional problem presented in Ref. 29. We first consider the potential distribution around a single isolated particle. 


\section{A. Potential distribution about a single particle}

\section{Diffuse-charge boundary layer}

In the singular thin-double-layer limit $\delta \ll 1$, a thin diffuse-charge layer of thickness $O(\delta)$ forms about the particle boundary. The leading-order boundary-layer solution of (7), in conjunction with (8), and attenuation at distances $\gg \delta$ from the boundary, is well known [38-40]. Expanding the potential in this domain as $\varphi \sim \Psi+O(\delta)$, one finds (dropping the $i$-subscript for now)

$$
\tanh \frac{\Psi}{4}=e^{-l / \delta} \tanh \frac{\zeta}{4} .
$$

Here $l$ is the normal distance from the surface (here assumed $O(\delta)$ ), and $\zeta$ is the $\varphi^{*}$ normalised leading-order voltage across the layer. This solution is compatible with either of the boundary conditions (8); $\zeta$ is asymptotic to the surface potential $\psi$, and related to the surface charge $\sigma$ by the Gouy-Chapman relation $\sigma=2 \sinh (\zeta / 2)$. It is well known that this solution is also compatible with more general "charge regulation"-type conditions [17, 41]. Thus in the present limit we may simply regard $\zeta$ as the prescribed surface property.

\section{Bulk domain}

The exponential decay of the Debye-scale potential,

$$
\Psi \sim 4 e^{-l / \delta} \tanh \frac{\zeta}{4} \quad \text { as } \quad l / \delta \rightarrow \infty,
$$

implies that the potential in the bulk domain (distances $\gg \delta$ from the particle boundary) is exponentially small in $\delta$. Hence, the bulk potential is governed by the linearized PB equation

$$
\delta^{2} \nabla^{2} \varphi \sim \varphi
$$

The form of Eq. (14) suggests expanding $\varphi$ in the form of a WKBJ ansatz [42, 43]

$$
\varphi \sim[A(\mathbf{x})+O(\delta)] e^{-u(\mathbf{x}) / \delta} .
$$

From (13), asymptotic matching with the diffuse-charge layer requires that

$$
u \sim l, \quad A \sim 4 \tanh \frac{\zeta}{4},
$$

as the particle boundary is approached $(l \ll 1)$. 
Substitution of (15) into (14) yields at leading order the "eikonal" equation

$$
|\nabla u|^{2}=1
$$

and at first order the "transport" equation

$$
\nabla u \cdot \nabla A=-\frac{A}{2} \nabla^{2} u
$$

Equations (17) and (18), in conjunction with the matching conditions (16), allow us to sequentially calculate the "phase" $u(\mathbf{x})$ and "amplitude" $A(\mathbf{x})$. The solution for the phase follows from a trivial application of Charpit's method of characteristics [44], or just by observation: $u(\mathbf{x})=l, l$ being the minimum distance of $\mathbf{x}$ from the particle boundary. Explicitly, if the particle boundary and outward normal are respectively parametrized as $\mathbf{x}=\mathbf{x}_{p}(\vec{s})$ and $\hat{\mathbf{n}}_{p}(\vec{s})$, then a parametric representation of the solution is

$$
u(\vec{s}, l)=l \quad \text { on } \quad \mathbf{x}(\vec{s}, l)=\mathbf{x}_{p}(\vec{s})+l \hat{\mathbf{n}}_{p}(\vec{s}) .
$$

We may say that at any given boundary point, a straight ray emanates in the direction of the local outward normal (since the particle is convex and smooth, there is a unique ray passing through any point $\mathbf{x}$ ).

Consider next the amplitude $A$. Noting that $\boldsymbol{\nabla} u=\hat{\mathbf{n}}_{p}(\vec{s})$, the transport equation (18) can be written as

$$
\nabla \cdot\left(A^{2} \hat{\mathbf{n}}_{p}\right)=0
$$

Considering an infinitesimal tube of rays $[45,46],(20)$ implies that $A^{2} / k$ remains constant along a ray, $k$ being the Gaussian curvature of the constant- $l$ 'wave front'. This result is entirely analogous to the light-intensity attenuation law of geometrical optics, from where we borrowed the phase-amplitude jargon. Now, as $l \rightarrow 0$, the matching condition (16) yields $A \rightarrow 4 \tanh (\zeta / 4)$, while the ray structure (19) implies that $k \rightarrow K$, where

$$
K\left(\rho_{1}, \rho_{2}\right)=\frac{1}{\rho_{1}(\vec{s}) \rho_{2}(\vec{s})}
$$

is the local Gaussian curvature of the particle boundary, $\rho_{1}$ and $\rho_{2}$ being the corresponding principal radii of curvature. It readily follows that

$$
A(\vec{s}, l)=4 \tanh \frac{\zeta}{4} \sqrt{\frac{K\left(\rho_{1}(\vec{s})+l, \rho_{2}(\vec{s})+l\right)}{K\left(\rho_{1}(\vec{s}), \rho_{2}(\vec{s})\right)}} .
$$




\section{B. Potential distribution in the bulk separating two particles}

We return now to the original problem of two convex particles $i=1,2$, with the separation satisfying $h \gg \delta$. The superposition approximation is applicable in this limit: the potential distribution is obtained by adding the distributions associated with each particle separately. Specifically, in the bulk domain we add together two ray solutions of the form (15), the ray

and particle-boundary parameters of particle $i$ being denoted as $\vec{s}_{i}, l_{i}, \hat{\mathbf{n}}_{p i}, \rho_{1 i}, \rho_{2 i}$, and $\zeta_{i}$. The bulk potential therefore reads

$$
\varphi \sim A_{1}\left(\vec{s}_{1}, l_{1}\right) e^{-l_{1} / \delta}+A_{2}\left(\vec{s}_{2}, l_{2}\right) e^{-l_{2} / \delta},
$$

with [cf. (22)]

$$
A_{i}\left(\vec{s}_{i}, l_{i}\right)=4 \tanh \frac{\zeta_{i}}{4} \sqrt{\frac{K\left(\rho_{i 1}\left(\vec{s}_{i}\right)+l_{i}, \rho_{2 i}\left(\vec{s}_{i}\right)+l_{i}\right)}{K\left(\rho_{i 1}\left(\vec{s}_{i}\right), \rho_{i 2}\left(\vec{s}_{i}\right)\right)}} .
$$

It is clear that, in an asymptotic sense, the two solutions forming (23) separately satisfy the bulk equation (14). Also, because of the exponential decay, rays emanating from one particle do not interfere with matching at the edge of the diffuse-layer surrounding the second particle. The overall structure of the solution is depicted in Fig. 2.

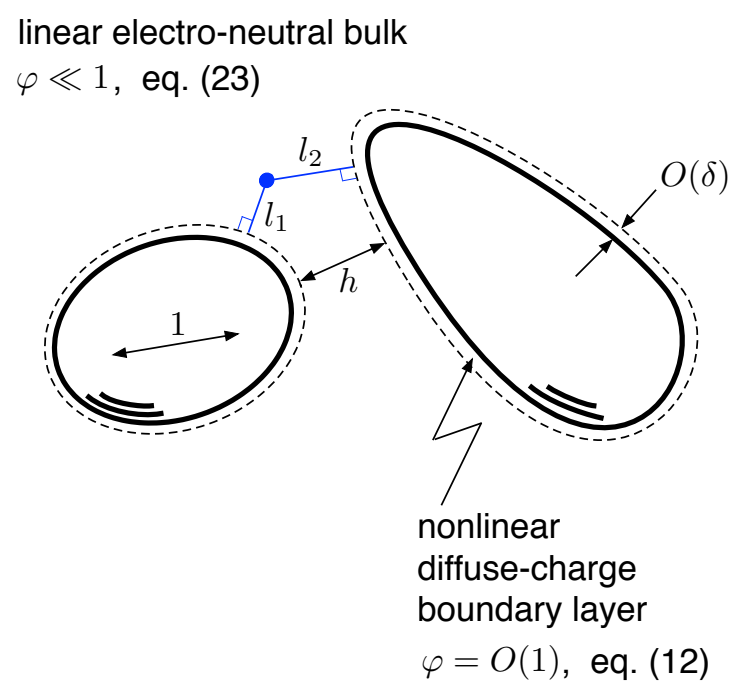

FIG. 2: Asymptotic structure of the potential distribution in the ray limit of §III. 


\section{The interaction force}

We shall now employ the parametric solution (23) towards calculating the interaction force. To this end, we deform the integration surface in (9) outward from the particle boundary into the bulk. In the latter domain, where the potential is exponentially small in $\delta$, we may expand the hydrostatic pressure (11) in powers of $\varphi$; the stress tensor (10) becomes

$$
\mathrm{T} \sim \boldsymbol{\nabla} \varphi \boldsymbol{\nabla} \varphi-\left(\frac{1}{2}|\boldsymbol{\nabla} \varphi|^{2}+\frac{1}{2} \varphi^{2} / \delta^{2}\right) \mathrm{I} .
$$

We see from (25) that, while the contributions to the bulk potential "carried" by the two families of rays superpose, the same does not apply for the bulk stress (25), which is quadratic in the potential. When substituting (23) into (25), noting that

$$
\nabla \varphi \sim-\delta^{-1}\left(\hat{\mathbf{n}}_{p 1} A_{1} e^{-l_{1} / \delta}+\hat{\mathbf{n}}_{p 2} A_{2} e^{-l_{2} / \delta}\right)
$$

we find three types of stress terms: (a) those associated with interaction of rays from particle 1 with rays from particles 2; (b \& c) those associated with self interaction of the rays from a single particle, either 1 or 2 . Terms of type (a)-(c) are respectively of order $\delta^{-2} e^{-\left(l_{1}+l_{2}\right) / \delta}, \delta^{-2} e^{-2 l_{1} / \delta}$, and $\delta^{-2} e^{-2 l_{2} / \delta}$, and so their magnitudes immensely vary with position, and their asymptotic hierarchy is spatially nonuniform. To overcome this apparent difficulty, we further specify the integration surface in (9) so as to make all three estimates comparable. Such a surface, for which $l_{1}=l_{2}$, is provided by the so-called "medial" surface generated by the centers of all spheres maximally inscribed between the two particle boundaries, see Fig. 6. Clearly, for any given point on this surface, $l_{1}=l_{2}=r$, where $r$ is the radius of the inscribed sphere centered at that point. Indeed, on this special surface the leading stress is

$$
\begin{aligned}
\mathbf{T} \sim \delta^{-2} e^{-2 r / \delta}\left\{-\left[A_{1}^{2}+A_{2}^{2}+\left(1+\hat{\mathbf{n}}_{p 1} \cdot \hat{\mathbf{n}}_{p 2}\right) A_{1} A_{2}\right] \mathbf{I}+A_{1}^{2} \hat{\mathbf{n}}_{p 1} \hat{\mathbf{n}}_{p 1}+\right. \\
\left.A_{1} A_{2}\left(\hat{\mathbf{n}}_{p 1} \hat{\mathbf{n}}_{p 2}+\hat{\mathbf{n}}_{p 2} \hat{\mathbf{n}}_{p 1}\right)+A_{2}^{2} \hat{\mathbf{n}}_{p 2} \hat{\mathbf{n}}_{p 2}\right\} .
\end{aligned}
$$

Eq. (27) shows that on the medial surface stress exponentially decays away from the center of the smallest maximally inscribed sphere (whose radius is $h / 2$ ). This enables extracting the dominant contribution to (9) via Laplace's method of integration.

As a preliminary step, we grid the medial surface with orthogonal curvilinear coordinates 
$\xi, \eta$ of unity metric, so that

$$
\hat{\mathbf{e}}_{\xi}=\frac{\partial \mathbf{x}}{\partial \xi}, \quad \hat{\mathbf{e}}_{\eta}=\frac{\partial \mathbf{x}}{\partial \eta}
$$

are orthogonal unit vectors. We further specify that $\xi, \eta=0$ coincides with the center of the smallest maximally inscribed sphere, hence $r(\xi=0, \eta=0)=h / 2$. As noted, the stress (27) exponentially decays away from this center. The latter can be made explicit via the expansion

$$
r \sim \frac{h}{2}+\frac{1}{2}\left(\frac{\partial^{2} r}{\partial \xi^{2}}\right)_{\mathbf{0}} \xi^{2}+\left(\frac{\partial^{2} r}{\partial \xi \partial \eta}\right)_{\mathbf{0}} \xi \eta+\frac{1}{2}\left(\frac{\partial^{2} r}{\partial \eta^{2}}\right)_{\mathbf{0}} \eta^{2}+\cdots
$$

the $\mathbf{0}$-subscript denoting evaluation at $\xi, \eta=0$. Expansion (29) makes use of the fact that $r$ is critical at $\xi, \eta=0$. Since the latter extremum is a minimum, and hence not a saddle point, the Hessian discriminant

$$
\Delta^{2}=\left(\frac{\partial^{2} r}{\partial \xi^{2}} \frac{\partial^{2} r}{\partial \eta^{2}}-\frac{\partial^{2} r}{\partial \xi \partial \eta}\right)_{\mathbf{0}}
$$

is positive. (This discriminant is invariant to the particular orientation of $\hat{\mathbf{e}}_{\xi}, \hat{\mathbf{e}}_{\eta}$ at $\mathbf{0}$, which we do not specify here.) In appendix $\mathrm{C}$ we study the medial surface near the point $\mathbf{0}$, finding the purely geometric result

$$
\begin{aligned}
4 \Delta^{2}=\left(\frac{1}{R_{11}+h / 2}\right. & \left.+\frac{1}{R_{21}+h / 2}\right)\left(\frac{1}{R_{12}+h / 2}+\frac{1}{R_{22}+h / 2}\right) \\
& +\left(\frac{1}{R_{11}+h / 2}-\frac{1}{R_{12}+h / 2}\right)\left(\frac{1}{R_{21}+h / 2}-\frac{1}{R_{22}+h / 2}\right) \sin ^{2} \phi
\end{aligned}
$$

where $R_{i j}$ denotes the value of $\rho_{i j}$ at minimum separation. Note that $R_{i j}$ is the dimensionless counterpart of $R_{i j}^{*}$ defined in the introduction (along with the orientation angle $\phi$ ). On the $O(\delta)$ scale, on which the exponential pre-factor in (27) decays, the remaining multiplicative part of the force integrand $\hat{\mathbf{n}} \cdot T$ remains approximately constant. Thus, for the latter part we require only the limiting value at minimum separation. Considering for example the force on particle 1 , the outward normal $\hat{\mathbf{n}}$ to the integration surface is $\sim \hat{\mathbf{k}}$, a unit vector parallel to the line of minimal distance connecting particle 1 to 2 . Similarly,

$$
\hat{\mathbf{n}}_{p 1} \sim \hat{\mathbf{k}}, \quad \hat{\mathbf{n}}_{p 2} \sim-\hat{\mathbf{k}}
$$

and

$$
A_{i} \sim\left(A_{i}\right)_{\mathbf{0}}=4 \tanh \frac{\zeta_{i}}{4} \sqrt{\frac{K\left(R_{i 1}+h / 2, R_{i 2}+h / 2\right)}{K\left(R_{i 1}, R_{i 2}\right)}} .
$$


We are now in the position of applying the two-dimensional version of Laplace's method. Substituting the above approximations into the integrand $\hat{\mathbf{n}} \cdot T$, and performing the resulting two-dimensional Gaussian quadrature [see Eq. (8.3.52) of Ref. 47], we find

$$
\mathbf{f}_{1} \sim \int \hat{\mathbf{n}} \cdot \mathrm{T} d \xi d \eta \sim-2 \pi \hat{\mathbf{k}} \frac{\left(A_{1}\right)_{\mathbf{0}}\left(A_{2}\right)_{\mathbf{0}}}{\Delta} \delta^{-1} e^{-h / \delta}
$$

We can simplify this result by noting that $2 \Delta=1 /[R]$, where $R$ denotes the effective Derjaguin radius (3) normalised by the characteristic length $a^{*}$, and [.] is the operator of adding $h / 2$ to all radii of curvature. Thus, the magnitude of the leading-order interaction force acting along the minimum-distance line between the particles reads

$$
F \sim 64 \pi \tanh \frac{\zeta_{1}}{4} \tanh \frac{\zeta_{2}}{4}[R] \sqrt{\frac{\left[K_{1}\right]\left[K_{2}\right]}{K_{1} K_{2}}} \delta^{-1} e^{-h / \delta}
$$

with $K_{i}=K\left(R_{i 1}, R_{i 2}\right)$, and with $F>0$ corresponding to a repulsive force.

\section{GENERALIZED DERJAGUIN APPROXIMATION}

The new closed-form approximation (35) is asymptotic in the limit $\delta \ll 1, h$. As outlined in the introduction, the latter domain of validity overlaps with that of the classical Derjaguin approximation, which is $\delta, h \ll 1$ (see Fig. 1). This suggests forming a uniformly valid approximation that is valid for all separations $h$ (as long as $\delta \ll 1$ ); the form of the new uniform approximation turns out to be remarkably simple.

In our dimensionless notation, Derjaguin's mapping (1) becomes

$$
F \sim 2 \pi R \int_{h}^{\infty} \Pi\left(h^{\prime}\right) d h^{\prime}
$$

To make the paper self-contained, we derive this well-established formula in appendix C, along with the effectively one-dimensional problem governing $\Pi$; we also give some of the standard approximations for $\Pi$ valid under various circumstances. Our derivation may appear different from the usual energy-based arguments [48], and it shows how (36) arises naturally from a field description in the appropriate asymptotic limit.

In the overlap domain, where $\delta \ll h$, $\Pi$ may be approximated by (B14). Derjaguin's mapping (36) then yields the closed-form approximation

$$
F \sim 64 \pi R \tanh \frac{\zeta_{1}}{4} \tanh \frac{\zeta_{2}}{4} \delta^{-1} e^{-h / \delta}
$$


which is quite often employed in practice due to its simplicity. Unsurprisingly, this is consistent with the $h \ll 1$ limit of (35). All we need is a generalisation of (36) such that it remains the same for $\delta, h \ll 1$, but degenerates in the limit $\delta \ll h$ to (35) instead of (37). Evidently, the required generalisation of (36) is

$$
F \sim 2 \pi[R] \sqrt{\frac{\left[K_{1}\right]\left[K_{2}\right]}{K_{1} K_{2}}} \int_{h}^{\infty} \Pi\left(h^{\prime}\right) d h^{\prime},
$$

which corresponds to the dimensional transformation (4) stated in the introduction. For later reference we note that, in the special case of two spheres $\left(R_{i 1}=R_{i 2}=R_{i}\right)$, the generalized Derjaguin radius simplifies to

$$
\text { two spheres: }[R] \sqrt{\frac{\left[K_{1}\right]\left[K_{2}\right]}{K_{1} K_{2}}}=\frac{R_{1} R_{2}}{R_{1}+R_{2}+h} \text {. }
$$

\section{COMPARISON WITH NUMERICAL SOLUTIONS}

In this section we compare the new ray-theory approximation (35) and the generalized Derjaguin approximation (38) to numerical solutions of the nonlinear PB formulation of §II. Following the methods of Refs. 30-32, we obtain numerical solutions for pairs of non-identical spheres by discretising the governing equation and boundary conditions using second-order finite-differences in a bi-spherical coordinate system, and solving the resulting set of nonlinear algebraic equations by means of a Newton-Raphson method. The interaction force is then obtained by discretising (9).

Figures 3 (fixed-charge conditions) and 4 (fixed-potential conditions) show results for pairs of non-identical spheres. We choose the characteristic length $a^{*}$ to be the radius of

particle 1 , and consider the case $R_{1}=1$ and $R_{2}=2$. The normalised surface properties are taken as $\left(\sigma_{1}=3, \sigma_{2}=1\right)$ in the fixed-charge case, and $\left(\psi_{1}=3, \psi_{2}=1\right)$ in the fixed-potential case. The dimensionless Debye length is taken to be $\delta=0.05$. We plot the interaction force $F$ divided by the scaling $\delta^{-1} e^{-h / \delta}$; we show the numerical solution (symbols), Eq. (36) — the classical Derjaguin approximation (dashed line), Eq. (35) — the new closed-form ray-theory approximation (dashed-dot line), and Eq. (38) the generalized uniformly valid Derjaguin approximation (solid lines). It is seen that the ray approximation (35) is highly accurate when the separation is just two or three times the Debye thickness, and that the uniform approximation is highly accurate at all separations. In order to draw the Derjaguin approximation, the one-dimensional problem governing $\Pi$ was solved without approximation (see 


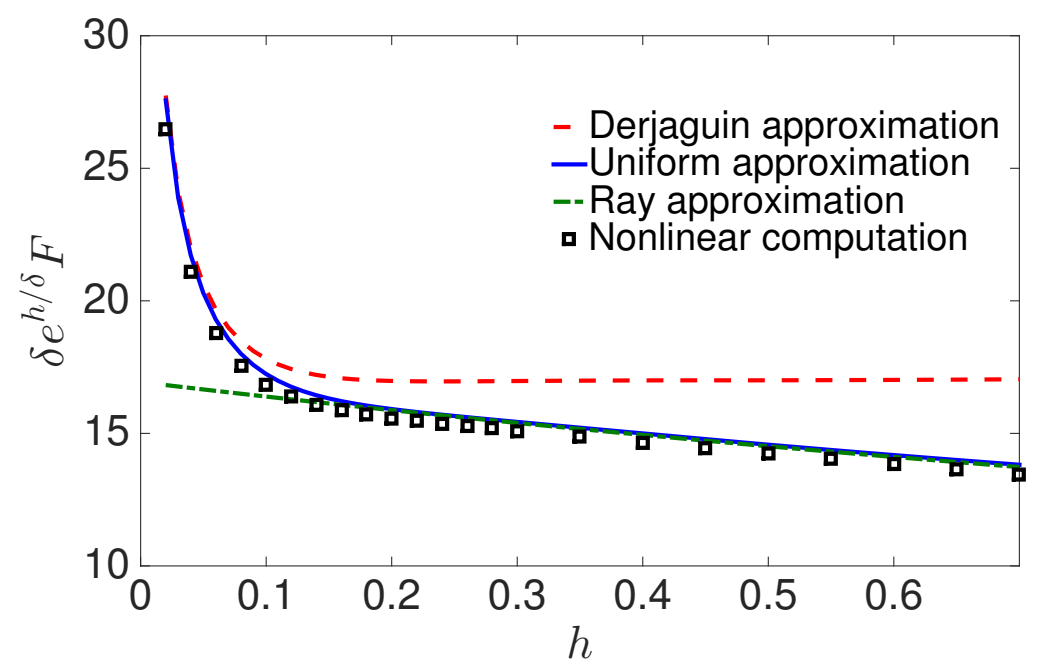

FIG. 3: Scaled interaction force as a function of dimensionless separation $h$ between a pair of fixed-charge spheres of dimensionless radii $R_{1}=1, R_{2}=2$, surface charge $\sigma_{1}=3, \sigma_{2}=1$, and dimensionless Debye thickness $\delta=0.05$. For details on the numerical solution and the various approximations depicted, see $\S \mathrm{V}$.

appendice $\S \mathrm{B})$. We note that the popular formula (37), visibly corresponding in the figures to the straight asymptote of the classical Derjaguin approximation, is strictly speaking only valid for $\delta \ll h \ll 1$, a very narrow domain.

The ray approximation (35) asymptotes to the "exact" interaction force as $\delta \rightarrow 0$, as long as $h \gg \delta$. To demonstrate this, we plot in Fig. 5 the ray approximation vs. numerical solutions for $\delta=0.2,0.1$ and 0.05 . For this figure, we consider two spheres having the same radius, with $\sigma_{1}=0.5$ and $\sigma_{2}=2$. To avoid clutter, the classical and generalized Derjaguin approximations are not shown.

\section{CONCLUDING REMARKS}

The generalized "effective radius" (4) extends Derjaguin's approximation to all interparticle separations. Our derivation allows for arbitrary three-dimensional convex surfaces. Since it is also not limited to small potentials relative to the thermal voltage, the single remaining condition is that the Debye length be small compared to characteristic particle size. A well known property of Derjaguin's approximation is that it depends only on properties of the surfaces at minimum separation. Somewhat surprisingly, we find that this is 
also the case beyond close proximity - this is a consequence of the exponential nature of EDL interactions. We find it remarkable that notwithstanding the increased generality, the new uniformly valid approximation (38) retains the form of Derjaguin's approximation (36), coming at almost no cost with respect to complexity.

Our main effort has been to derive the new closed-form approximation (35) in the limit where the Debye length is small compared to both the separation and the characteristic particle size. The generalized Derjaguin mapping then emerged naturally by noting the overlap in the domains of validity of the classical Derjaguin approximation and the new closed-form approximation. Our analysis builds on the ray-theory approach put forward in Ref. 29 within the context of planar geometries. A subtle point is that the two-dimensional ray-theory analysis is not included in the present three-dimensional one. This is because the stress in Ref. 29 is localised about a line, while here it is localised about a point; crucially, this leads to a different scaling of the interaction force with $\delta$.

The interaction force is asymptotically small in the domain of validity of the ray approximation (35). The latter domain is also where the new uniform approximation (38) improves on the classical Derjaguin approximation (36). The importance of the interaction at a given separation, however, is not judged in comparison with its magnitude at a hypothetical smaller separation. The question, rather, is how big are the other forces in a given physical scenario? As mentioned in the introduction, it is often the case that the separation - although determined by a balance between EDL and, e.g., gravity or van der

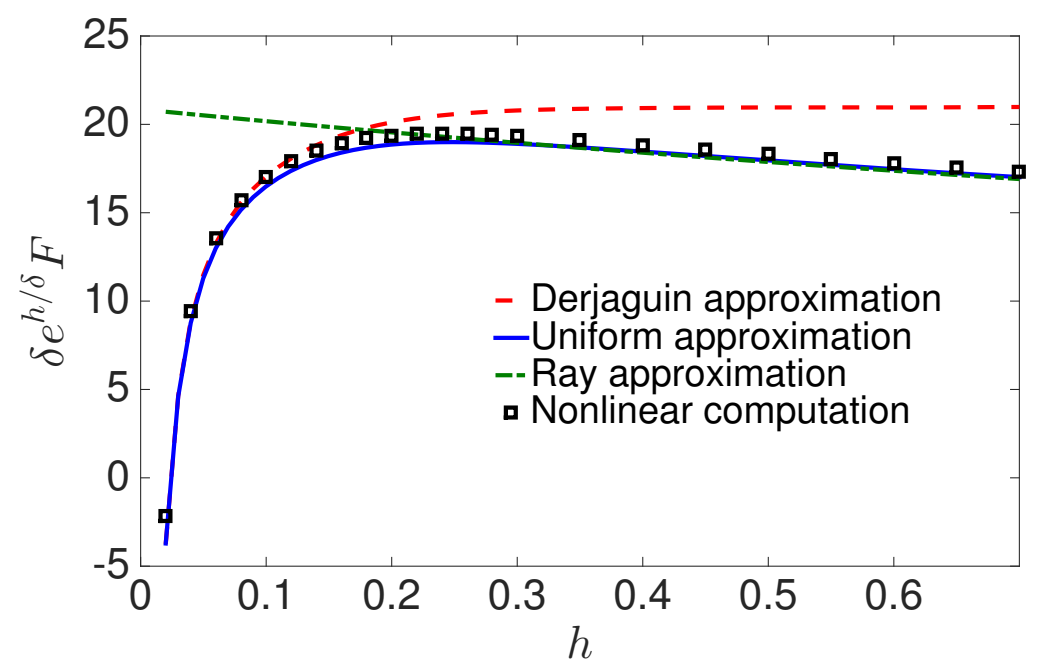

FIG. 4: Same as Fig. 3 but for fixed-potential spheres, with $\psi_{1}=3$ and $\psi_{2}=1$. 
Waals forces - is several times the Debye length. In that domain, the relative difference between the classical and generalised Derjaguin approximation is $O(1)$. Admittedly, while Derjaguin's classical approximation is not asymptotically valid at these separations, it provides an approximation which is probably sufficiently accurate from a practical point of view.

Our results hold if one of the particles is replaced by a plane wall. Note however that then the dependence of the effective radius on $h$ vanishes, and our result coincides with the classical Derjaguin approximation. The fortuitous improved accuracy of Derjaguin's approximation for the particle-plane configuration was found also in the two-dimensional case [29], and in previous numerical simulations [31]. We also note that our results remain applicable even if the zeta potential varies on the characteristic particle scale [29]. In (23), the amplitudes $A_{i}$ would depend on $\vec{s}_{i}$ not only through surface geometry, but also through $\zeta_{i}\left(\vec{s}_{i}\right)$. The result for the leading-order force (35), however, would remain the same, with $\zeta_{i}$ evaluated at minimum separation. Further clarification is due regarding our ab initio neglect of the electric field within the solid particles. This is valid (except for particles with an exceptionally large dielectric constant) because all of our results pertain to the limit $\delta \ll 1$ where the electric displacement in the Debye layers is $O(1 / \delta)$ compared to that in

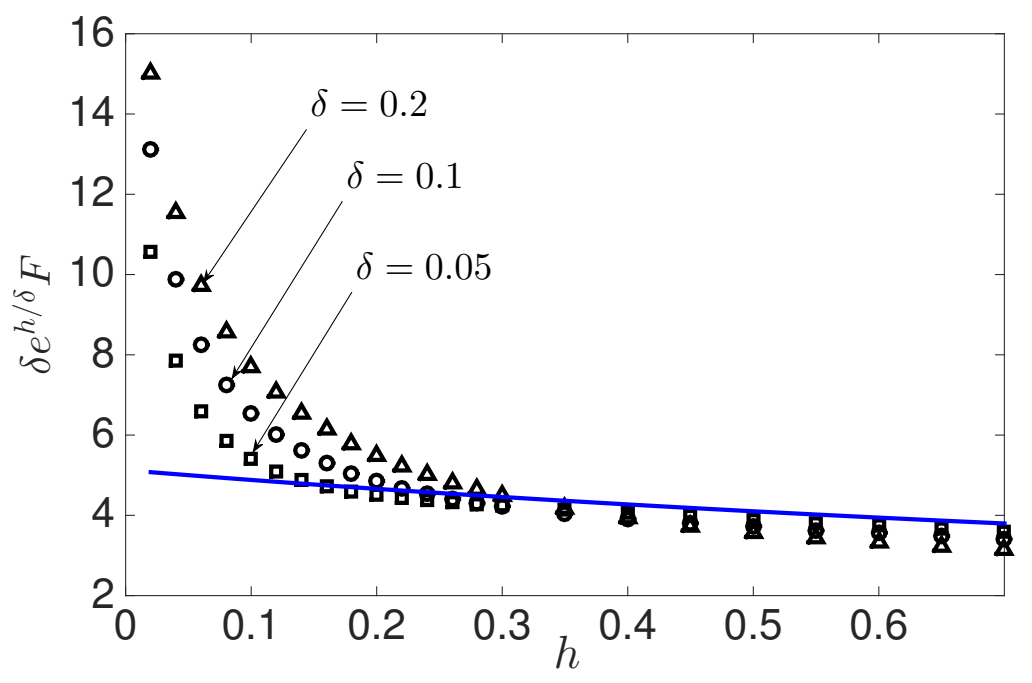

FIG. 5: Convergence of the ray approximation (35) (blue line) to numerical solutions (symbols) as $\delta \rightarrow 0$, under the condition $h \gg \delta$. The results shown are for a pair of spheres of equal radius $\left(R_{1}=R_{2}=1\right)$, and fixed charge conditions $\left(\sigma_{1}=0.5, \sigma_{2}=2\right)$. 
the solids.

Our extension to Derjaguin's approximation relies on a continuum-level description, specifically the nonlinear PB model for a dilute binary symmetric electrolyte. The PB formulation has proved successful in describing simple scenarios, including those involving multivalent electrolytes [49] (it would be fairly straightforward to generalize our analysis to this case). Nevertheless, there are many studies aimed at providing more detailed descriptions [50-52], either via molecular-dynamics simulations, density functional theory, or various modified continuum descriptions. The latter models attempt to take into account non-ideal characteristics of the electrolyte solution, such as steric effects and ion-ion electrostatic correlations. Clearly, those which degenerate to the linear PB equation in the bulk [53] would be amenable to our ray-theory approach. Indeed, the modification to the bulk potential would enter only through the value of the amplitude $A$ obtained from matching [cf. (16)]; since this modification would be anyway captured by the one-dimensional $\Pi$ function the generalized Derjaguin approximation (38) would hold as is. Otherwise, our ray-approach would have to be modified. This is certain with respect to recent models of highly concentrated electrolytes and room-temperature ionic liquids [54-57]. Even in the bulk, or for low potentials, these involve a fourth-order PDE, in some cases predicting an oscillating exponential attenuation of the diffuse-layer potential.

Finally, it is well known that Derjaguin's close-proximity approximation is not uniquely applicable to EDL interactions. In fact, it is employed on a regular basis to study many other surface-surface interactions, such as van der Waals and depletion forces. Beyond close proximity, the general requirement is for the relevant interaction length scale to be $\ll R^{*}$. In contrast, our generalisation of Derjaguin's mapping to arbitrary separations strongly relies on the exponential decay of the EDL interaction, and hence it might only be possible to adopt it to other exponential interactions (e.g. nuclear forces, or opto-mechanical configurations involving evanescent waves). Clearly, a comparable generalization for algebraically decaying interactions, if at all possible, would necessarily involve also surface properties away from minimum separation. 


\section{Appendix A: Local system of coordinates}

We describe here a cartesian coordinate system $(x, y, z)$, with unit vectors $\left(\hat{\mathbf{e}}_{x}, \hat{\mathbf{e}}_{y}, \hat{\mathbf{e}}_{z}\right)$, that will be used in the next two appendices (as in the text, lengths are normalised by the reference scale $\left.a^{*}\right)$. We place the origin at the middle of the minimum-length $(=h)$ line segment connecting the particle boundaries, with the unit vector $\hat{\mathbf{e}}_{z}$ pointing towards 'surface 1' along this line, see Fig. 6. By construction, the tangent planes at the points

of contact of the $z$ axis and the two boundaries are both parallel to the $x-y$ plane; thus locally the two surfaces are described by osculating paraboloids with $\hat{\mathbf{e}}_{z}$ as their common axis. To fully specify the system, we orient $\hat{\mathbf{e}}_{x}$ and $\hat{\mathbf{e}}_{y}$ such that they respectively point in the directions of the maximum and minimum curvature lines of the osculating paraboloid of boundary 1.

It follows that, to second order in $x$ and $y$, surfaces 1 and 2 respectively read

$$
g_{1}(x, y) \sim \frac{h}{2}+\frac{x^{2}}{2 R_{11}}+\frac{y^{2}}{2 R_{12}}+\cdots, \quad g_{2}(x, y) \sim-\frac{h}{2}-\frac{x^{\prime 2}}{2 R_{21}}-\frac{y^{\prime 2}}{2 R_{22}}+\cdots
$$

where

$$
x^{\prime}=x \cos \phi+y \sin \phi, \quad y^{\prime}=-x \sin \phi+y \cos \phi,
$$

$\phi$ denoting the angle in the $x-y$ plane between the principal curvature bases of the two osculating paraboloids. In constructing the (classical) Derjaguin approximation, the difference between the two surfaces is the geometrically important quantity. Clearly this difference is, locally, also a paraboloid about the $z$ axis. In a coordinate system $(\dot{x}, y, z)$ aligned with the principal basis of the latter paraboloid, it can be locally approximated as

$$
g_{1}-g_{2} \sim h+\frac{\lambda_{1}}{2} \dot{x}^{2}+\frac{\lambda_{2}}{2} \dot{y}^{2}+\cdots
$$

with the constants $\lambda_{1}, \lambda_{2}$ satisfying the relation [26]

$$
\lambda_{1} \lambda_{2}=\left(\frac{1}{R_{11}}+\frac{1}{R_{21}}\right)\left(\frac{1}{R_{12}}+\frac{1}{R_{22}}\right)+\left(\frac{1}{R_{11}}-\frac{1}{R_{12}}\right)\left(\frac{1}{R_{21}}-\frac{1}{R_{22}}\right) \sin ^{2} \phi
$$

this expression can be identified as $1 / R^{2}$, where $R=R^{*} / a^{*}$ is the normalised effective Derjaguin radius [cf. (3)]. 


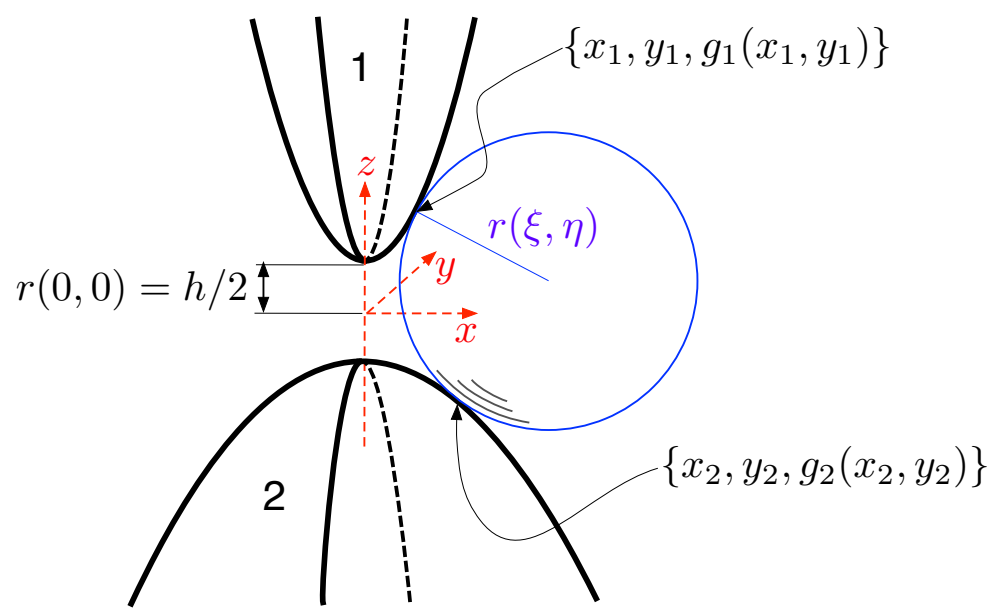

FIG. 6: Local coordinate system referred to in appendices A-C. Also shown is one of the maximally inscribed spheres whose center lies on the medial integration surface.

\section{Appendix B: The limit $\delta, h \ll 1$ with $h \sim O(\delta)$ : Derjaguin's approximation}

We consider the gap region using the stretched "difference" coordinates [cf. (A3)],

$$
X=\dot{x} / h^{1 / 2}, \quad Y=\dot{y} / h^{1 / 2}, \quad Z=z / h,
$$

whereby surfaces 1 and 2 may be respectively approximated as

$$
Z \sim G_{1}(X, Y)+O(h), \quad Z \sim G_{2}(X, Y)+O(h)
$$

we shall only make use of the difference $G_{1}-G_{2}$, which is

$$
D(X, Y)=1+\frac{\lambda_{1}}{2} X^{2}+\frac{\lambda_{2}}{2} Y^{2}
$$

With the above scalings, together with the potential expansion $\varphi \sim \Phi+O(h)$, we find from (7) the leading-order equation

$$
\frac{\delta^{2}}{h^{2}} \frac{\partial^{2} \Phi}{\partial Z^{2}}=\sinh \Phi
$$

and from (8) the supplementary conditions

$$
\Phi=\psi_{1} \quad \text { or } \quad \frac{\delta}{h} \frac{\partial \Phi}{\partial Z}=\sigma_{1} \quad \text { at } \quad Z=G_{1}(X, Y)
$$

and

$$
\Phi=\psi_{2} \quad \text { or } \quad \frac{\delta}{h} \frac{\partial \Phi}{\partial Z}=-\sigma_{2} \quad \text { at } \quad Z=G_{2}(X, Y)
$$


The stress tensor (10) is, to leading order,

$$
\mathrm{T} \sim-\hat{\mathbf{e}}_{z} \hat{\mathbf{e}}_{z} \tilde{\Pi}(D)
$$

where

$$
\tilde{\Pi}(D)=\frac{1}{\delta^{2}}(\cosh \Phi-1)-\frac{1}{2 h^{2}}\left(\frac{\partial \Phi}{\partial Z}\right)^{2}
$$

is, from (B4), independent of $Z$, and depends on $X, Y$ only through the separation $D$. Thanks to the fast decay of $\tilde{\Pi}(D)$ with growing $D$, the magnitude of the leading interaction force $(9)$ is

$$
F \sim h \int_{-\infty}^{\infty} \int_{-\infty}^{\infty} \tilde{\Pi}(D) d X d Y
$$

this force is directed in the $\hat{\mathbf{e}}_{z}$ direction $(F>0$ corresponds to a repulsive force). Following White [26], an obvious change of integration variables yields

$$
F \sim \frac{2 \pi h}{\sqrt{\lambda_{1} \lambda_{2}}} \int_{1}^{\infty} \tilde{\Pi}(D) d D
$$

Reverting to unscaled coordinates by defining $\Pi(x)=\tilde{\Pi}(x / h)$, and recalling (A4), we find Derjaguin's approximation in dimensionless form:

$$
F \sim 2 \pi R \int_{h}^{\infty} \Pi\left(h^{\prime}\right) d h^{\prime}
$$

Noting that $\Pi(h)=\tilde{\Pi}(1)$, to determine $\Pi(h)$ one simply needs to solve (B4), with the boundary conditions (B5) and (B6) applied at $Z=1$ and $Z=0$, respectively; the dependence on the factor $h / \delta$ enters through its appearance as a parameter in (B4). Exact solutions can be obtained in terms of special functions [58, 59], or numerically. There are also several approximations which are often employed [60]. For example, at low voltages with fixed-potential conditions, one finds

$$
\Pi(h) \sim \frac{\psi_{1}^{2}+\psi_{2}^{2}}{2 \delta^{2} \sinh ^{2}(h / \delta)}\left[\frac{2 \psi_{1} \psi_{2}}{\psi_{1}^{2}+\psi_{2}^{2}} \cosh (h / \delta)-1\right] .
$$

while for fixed-charge conditions,

$$
\Pi(h) \sim \frac{\sigma_{1}^{2}+\sigma_{2}^{2}}{2 \delta^{2} \sinh ^{2}(h / \delta)}\left[\frac{2 \sigma_{1} \sigma_{2}}{\sigma_{1}^{2}+\sigma_{2}^{2}} \cosh (h / \delta)+1\right] .
$$

At large separations compared with the Debye length, $\delta \ll h$, the above two expressions asymptotically coincide. At those separations, however, we have the generic thin-doublelayer approximation

$$
\Pi(h) \sim 32 \delta^{-2} \tanh \frac{\zeta_{1}}{4} \tanh \frac{\zeta_{2}}{4} e^{-h / \delta},
$$

which is not limited to low potentials. In (B14), $\zeta_{i}$ is $\psi_{i}$ in the fixed-potential case, or determined from $\sigma_{i}=2 \sinh \left(\zeta_{i} / 2\right)$ in the fixed-charge case. 


\section{Appendix C: The Hessian discriminant $\Delta$ of the radius function $r(\xi, \eta)$}

We here set out to calculate the Hessian discriminant $\Delta$ defined in (30) as a function of the geometry of the two surfaces and the separation $h$. From (29) we infer that to calculate $\Delta$ it is sufficient to determine the distribution of the inscribed-sphere radius $r$ to second order with respect to distances from the center of the smallest inscribed sphere.

We start by considering an arbitrarily chosen maximally inscribed sphere that is tangent to surface 1 at $\left\{x_{1}, y_{1}, g_{1}\left(x_{1}, y_{1}\right)\right\}$, and to surface 2 at $\left\{x_{2}, y_{2}, g_{2}\left(x_{2}, y_{2}\right)\right\}$ [see $\S \mathrm{A}$ and fig. 6]. For the present, let us consider the coordinate pairs $\left(x_{2}, y_{2}\right)$, and the center of the latter inscribed sphere $\mathbf{x}_{m}$ along with its radius $r$, as functions of the pair $\left(x_{1}, y_{1}\right)$; the required connection of this pair with the surface coordinates $(\xi, \eta)$ introduced in $\S$ III C will be made later. In particular, we define the functions

$$
x_{2}=x_{2}\left(x_{1}, y_{1}\right), \quad y_{2}=y_{2}\left(x_{1}, y_{1}\right), \quad r(\xi, \eta)=\tilde{r}\left(x_{1}, y_{1}\right), \quad \mathbf{x}_{m}(\xi, \eta)=\tilde{\mathbf{x}}_{m}\left(x_{1}, y_{1}\right),
$$

which may in principal be determined from the vector relations defining the medial surface:

$$
\begin{aligned}
\tilde{\mathbf{x}}_{m}\left(x_{1}, y_{1}\right)=x_{1} \hat{\mathbf{e}}_{x}+y_{1} \hat{\mathbf{e}}_{y}+g_{1}\left(x_{1}, y_{1}\right) \hat{\mathbf{e}}_{z}+\tilde{r}\left(x_{1}, y_{1}\right) \hat{\mathbf{n}}_{p 1}\left(x_{1}, y_{1}\right) \\
=x_{2} \hat{\mathbf{e}}_{x}+y_{2} \hat{\mathbf{e}}_{y}+g_{2}\left(x_{2}, y_{2}\right) \hat{\mathbf{e}}_{z}+\tilde{r}\left(x_{1}, y_{1}\right) \hat{\mathbf{n}}_{p 2}\left(x_{2}, y_{2}\right) .
\end{aligned}
$$

We solve (C2) only up to orders in $\left(x_{1}, y_{1}\right)$ needed for the exact calculation of $\Delta$. To this end, we substitute into (C2) expansions (A1), the associated expansions for the boundary normal vectors $\hat{\mathbf{n}}_{p 1}$ and $\hat{\mathbf{n}}_{p 2}$, and the Taylor-series expansion

$$
\tilde{r}\left(x_{1}, y_{1}\right) \sim \frac{h}{2}+\frac{1}{2}\left(\frac{\partial^{2} \tilde{r}}{\partial x_{1}^{2}}\right)_{\mathbf{0}} x_{1}^{2}+\left(\frac{\partial^{2} \tilde{r}}{\partial x_{1} \partial y_{1}}\right)_{\mathbf{0}} x_{1} y_{1}+\frac{1}{2}\left(\frac{\partial^{2} \tilde{r}}{\partial y_{1}^{2}}\right)_{\mathbf{0}} y_{1}^{2}+\cdots
$$

We next consider the $\hat{\mathbf{e}}_{x}, \hat{\mathbf{e}}_{y}$ components of the second equality in (C2). Comparing terms to first order yields $\left(x_{2}, y_{2}\right)$ to leading order in $\left(x_{1}, y_{1}\right)$. The $\hat{\mathbf{e}}_{z}$ component, which is nontrivial only at second order, then yields the second-order coefficients in expansion (C3). The resulting expressions are lengthy and are not given here.

The last and final step is to connect the second-order partial derivatives of $r$ with respect to $\left(x_{1}, y_{1}\right)$ appearing in $(\mathrm{C} 3)$ and those with respect to the surface coordinates $(\xi, \eta)$ appearing in (29). This is performed by applying the chain rule; since $r$ attains its minimum $h / 2$ at the origin, we require only the first-order partial derivatives relating $\left(x_{1}, y_{1}\right)$ and $(\xi, \eta)$. We find these by noting that the osculating paraboloid of the medial surface at the origin is 
tangent to the $x-y$ plane, and hence can be written as

$$
\mathbf{x}_{m}(\xi, \eta) \sim \xi \hat{\mathbf{e}}_{x}+\eta \hat{\mathbf{e}}_{y}+O\left(\xi^{2}, \eta^{2}, \xi \eta\right)
$$

without loss of generality, we make here a convenient $\Delta$-invariant choice for the orientation of $\hat{\mathbf{e}}_{\xi}$ and $\hat{\mathbf{e}}_{\eta}$ at the origin. Comparing (C4) with the first equality in (C2), we find

$$
\left(\frac{\partial x_{1}}{\partial \xi}\right)_{\mathbf{0}}=\left(1+\frac{h}{2 R_{11}}\right)^{-1}, \quad\left(\frac{\partial y_{1}}{\partial \eta}\right)_{\mathbf{0}}=\left(1+\frac{h}{2 R_{12}}\right)^{-1}, \quad\left(\frac{\partial x_{1}}{\partial \eta}\right)_{\mathbf{0}},\left(\frac{\partial y_{1}}{\partial \xi}\right)_{\mathbf{0}}=0 .
$$

Result (31) then follows.

\section{ACKNOWLEDGMENTS}

This research was supported in part by EPSRC Mathematics Platform grant EP/I019111/1.

[1] J. Lyklema, Fundamentals of Interface and Colloid Science, Vol. II (Academic, New York, 1995).

[2] D. F. Evans and H. Wennerström, The colloidal domain (Wiley-Vch New York, 1999).

[3] D. A. Saville, Annu. Rev. Fluid Mech. 9, 321 (1977).

[4] H. A. Stone, A. D. Stroock, and A. Ajdari, Annu. Rev. Fluid Mech. 36, 381 (2004).

[5] H. C. Chang and L. Y. Yeo, Electrokinetically driven microfluidics and nanofluidics (Cambridge University Press Cambridge, UK, 2010).

[6] B. J. Kirby, Micro-and nanoscale fluid mechanics: transport in microfluidic devices (Cambridge University Press, 2010).

[7] A. Revil, P. A. Pezard, and P. W. J. Glover, J. Geophys. Res. 104, 20021 (1999).

[8] J. Newman and K. E. Thomas-Alyea, Electrochemical systems (John Wiley \& Sons, 2012).

[9] I. Rubinstein, Electro-diffusion of ions, Vol. 11 (SIAM, 1990).

[10] S. G. Bike and D. C. Prieve, Int. J. Multiphase Flow 16, 727 (1990).

[11] E. J. W. Verwey and J. T. G. Overbeek, Theory of the Stability of Lyophobic Colloids (Elsevier, Amsterdam, 1948).

[12] D. C. Prieve, P. J. Sides, and C. L. Wirth, Curr. Opin. Colloid Interface Sci. 15, 160 (2010).

[13] C. L. Wirth, R. M. Rock, P. J. Sides, and D. C. Prieve, Langmuir 27, 9781 (2011). 
[14] H. H. Strey, V. A. Parsegian, and R. Podgornik, Phys. Rev. Lett. 78, 895 (1997).

[15] K. D. Dorfman, Rev. Mod. Phys. 82, 2903 (2010).

[16] P. A. Harvey, A. V. Nguyen, and G. M. Evans, J. Colloid Interface Sci. 250, 337 (2002).

[17] D. C. Prieve and E. Ruckenstein, J. Theor. Biol. 56, 205 (1976).

[18] M. Hermansson, Colloids Surf., B 14, 105 (1999).

[19] A. T. Poortinga, R. Bos, W. Norde, and H. J. Busscher, Surf. Sci. Rep. 47, 1 (2002).

[20] S. A. Edwards and D. R. M. Williams, Curr. Opin. Colloid In. 9, 139 (2004).

[21] T. J. Senden, C. J. Drummond, and P. Kekicheff, Langmuir 10, 358 (1994).

[22] S. G. Flicker and S. G. Bike, Langmuir 9, 257 (1993).

[23] J. Sotres and A. M. Baró, Biophys. J. 98, 1995 (2010).

[24] L. Guldbrand, B. Jonsson, H. Wennerstrom, and P. Linse, J. Chem. Phys 80, 2221 (1984).

[25] W. B. Russel, D. A. Saville, and W. R. Schowalter, Colloidal dispersions (Cambridge university press, 1992).

[26] L. R. White, J. Colloid Interface Sci. 95, 286 (1983).

[27] B. A. Todd and S. J. Eppell, Langmuir 20, 4892 (2004).

[28] R. S., R. P. C., P. G., and B. M., Phys. Chem. Chem. Phys. 8, 2531 (2006).

[29] O. Schnitzer, Phys. Rev. E 91, 022307 (2015).

[30] S. L. Carnie, D. Y. C. Chan, and J. Stankovich, J. Colloid Interface Sci. 165, 116 (1994).

[31] J. Stankovich and S. L. Carnie, Langmuir 12, 1453 (1996).

[32] P. Warszyński and Z. Adamczyk, J. Colloid Interface Sci. 187, 283 (1997).

[33] S. L. Carnie, D. Y. C. Chan, and J. S. Gunning, Langmuir 10, 2993 (1994).

[34] A. B. Glendinning and W. B. Russel, J. Colloid Interface Sci. 93, 95 (1983).

[35] L. N. McCartney and S. Levine, J. Colloid Interface Sci. 30, 345 (1969).

[36] G. M. Bell, S. Levine, and L. N. McCartney, J. Colloid Interface Sci. 33, 335 (1970).

[37] J. E. Sader, S. L. Carnie, and D. C. Chan, J. Colloid Interface Sci. 171, 46 (1995).

[38] I. Rubinstein and B. Zaltzman, Math. Models Methods Appl. Sci. 11, 263 (2001).

[39] E. Yariv, Chem. Eng. Commun. 197, 3 (2009).

[40] O. Schnitzer and E. Yariv, Phys. Rev. E 86, 021503 (2012).

[41] R. Pericet-Camara, G. Papastavrou, S. H. Behrens, and M. Borkovec, J. Phys. Chem. B 108, 19467 (2004).

[42] E. J. Hinch, Perturbation methods (Cambridge university press, 1991). 
[43] S. J. Chapman, J. M. H. Lawry, and J. R. Ockendon, SIAM J. Appl. Math 60, 121 (1999).

[44] J. R. Ockendon, S. Howison, A. Lacey, and A. Movchan, Applied partial differential equations (Oxford University Press, 2003).

[45] M. Born and E. Wolf, Principles of optics: electromagnetic theory of propagation, interference and diffraction of light (CUP Archive, 1999).

[46] M. H. Holmes, Introduction to perturbation methods, Vol. 20 (Springer Science \& Business Media, 2012).

[47] N. Bleistein and R. A. Handelsman, Asymptotic expansions of integrals (Courier Corporation, 1975).

[48] R. F. Probstein, Physicochemical hydrodynamics: an introduction (John Wiley \& Sons, 2005).

[49] R.-C. F. J. M., T. G., M. P., and B. M., Langmuir 30, 4551 (2014).

[50] P. Attard, D. J. Mitchell, and B. W. Ninham, J. Chem. Phys 88, 4987 (1988).

[51] P. Attard, D. J. Mitchell, and B. W. Ninham, J. Chem. Phys 89, 4358 (1988).

[52] M. Bostrom, D. R. M. Williams, and B. W. Ninham, Phys. Rev. Lett. 87, 168103 (2001).

[53] A. S. Khair and T. M. Squires, J. Fluid Mech. 640, 343 (2009).

[54] M. Z. Bazant, B. D. Storey, and A. A. Kornyshev, Phys. Rev. Lett. 106, 046102 (2011).

[55] S. Perkin, Phys. Chem. Chem. Phys. 14, 5052 (2012).

[56] D. Vella, S. Perkin, and A. Goriely, J. Chem. Phys 141, 094904 (2014).

[57] A. A. Lee, D. Vella, and A. Goriely, arXiv preprint arXiv:1502.01276 (2015).

[58] S. H. Behrens and M. Borkovec, Phys. Rev. E 60, 7040 (1999).

[59] P. M. Biesheuvel, J. Colloid Interface Sci. 275, 514 (2004).

[60] H. Ohshima, Electrical phenomena at interfaces and biointerfaces: fundamentals and applications in nano-, bio-, and environmental sciences (John Wiley \& Sons, 2012). 